\title{
Is the Truth Table Task Mistaken?
}

\author{
Aline Sevenants, Kristien Dieussaert and Walter Schaeken \\ University of Leuven
}

Keywords: conditionals, truth table task, induction problem, cognitive ability, mental models theory, suppositional theory

\section{Corresponding author:}

\author{
Aline Sevenants (Aline.Sevenants@ psy.kuleuven.be) \\ University of Leuven, Laboratory of Experimental Psychology \\ 102 Tiensestraat, \\ B-3000 Leuven, Belgium
}

Tel: $+32(0) 16 / 32.59 .52$

Fax: +32 (0) 16/32.60.99 


\section{Acknowledgments}

This research was carried out with financial support of the OT research project (3H110244) granted to Prof. Dr. Walter Schaeken. 


\begin{abstract}
There is ample evidence that in classical truth table task experiments false-antecedent are judged as 'irrelevant'. Instead of interpreting this in support of a suppositional representation of conditionals, Schroyens (2010a, 2010b) attributes it to the induction problem: the impossibility of establishing the truth of a universal claim on the basis of a single case. In the first experiment, a truth table task with four options is administered and the correlation with intelligence is inspected. It is observed that 'undetermined' is chosen in one third of the judgments and 'irrelevant' in another third. A positive correlation is revealed between intelligence and the number of 'irrelevant' and 'undetermined' judgments. The data do not exclude that a part of the 'irrelevant' judgments in classical truth table task experiments might be caused by the induction problem. In the second experiment, participants are presented with a simplified four-option truth table task and asked for a justification of their judgments. These justifications show the induction problem is not the reason for choosing the 'irrelevant' or 'undetermined' option, which is supportive for a suppositional representation of conditionals.
\end{abstract}




\section{Introduction}

The interest in the linguistic, psychological and logical meaning of 'if' has provided us with a long history of research on the mental representation of conditionals ('if $\underline{\text { Antecedent then }}$ Consequent' sentences, e.g., 'If the letter is $B$, then the number is 7 '). Combining the truth value of antecedent and consequent, four truth table cases arise: two true-antecedent cases (TT: ' $B$ ' and '7' and TF: ' $B$ ' and 'not 7') and two false-antecedent-cases (FT: 'not $B$ ' and ' 7 ' and FF: 'not $B$ ' and 'not 7 '). According to one psychological theory, 'if' is represented as the truth table for material implication of standard logic, meaning that only $\mathrm{TF}$ falsifies the conditional. An alternative logical possibility is the material equivalence: both TF and FT falsify the conditional. Wason (1966) was the first to introduce the 'defective' truth table (also known as 'three-valued' or 'de Finetti table', following de Finetti, 1967; Politzer, Over \& Baratgin, 2010), in which falseantecedent-cases (FT and FF) are considered as 'irrelevant' with respect to the conditional. Ever since, there has been substantial debate regarding the status of these false-antecedent-cases between the two main theories accounting for the mental representation of conditionals: the Mental Models Theory (MMT; Johnson-Laird \& Byrne, 1991, 2002) and the Suppositional Theory (ST; Evans, Handley, Neilens \& Over, 2007; Evans \& Over, 2004). More specifically, the observation that people represent conditionals as a 'defective' truth table has become a major point of contention. The ST states that false-antecedent-cases are 'irrelevant' to the truthjudgment of a conditional, whereas the MMT claims the conditional to be 'true' in these cases. In the truth table task, participants are classically asked to evaluate for each of the four cases (TT, TF, FT and FF) whether that situation 'makes the conditional rule true', 'makes the rule false' or 'is irrelevant with respect to the truth of the rule'. 
According to the ST, people evaluate conditionals by means of the Ramsey test. That is, they "hypothetically add $p$ to their stock of knowledge and evaluate their degree of belief in $q$ given p" (Ramsey, 1931/1990 p.247). So they first estimate the probability of the consequent and the antecedent occurring together (TT), and then the probability of the antecedent together with the non-occurrence of the consequent (TF). For the ones having disposal of sufficient cognitive resources to complete both stages, this leads to an estimation of the probability of occurrence of the consequent, given the antecedent. Running this Ramsey test, participants disregard falseantecedent-cases and only focus on those cases in which the antecedent is 'true', resulting in a 'defective' pattern.

According to the MMT however, reasoners construct mental models of the possibilities compatible with the premises, but they initially and by default do not represent what is false. Therefore, their conclusion is based on the initial model $([\mathrm{A}] \mathrm{C} \ldots)$. Under certain circumstances however, individuals make 'mental footnotes' about the falsity of clauses (represented by the ellipsis). If they are able to retain these footnotes, people can flesh out the implicitly represented information into fully explicit models, which represent clauses even when they have false antecedents. This in turn leads to 'logical' answer patterns on the truth table task (i.e., a 'true' judgment of the false-antecedent-cases) for those able to flesh out the initial model into fully explicit models. The ones lacking cognitive resources to flesh out give a 'defective' answerpattern (i.e., the false-antecedent-cases are judged to be 'irrelevant').

Lately there has been quite some experimental evidence (Barrouillet, 2008; Evans et al., 2007; Sevenants, Dieussaert \& Schaeken, 2011) for a positive correlation between intelligence and 'defective' answer patterns and even for a negative correlation between intelligence and 'logical' answer patterns. These findings contradict the claim of the MMT that the most intelligent participants are the ones giving a 'logical' answer-pattern. 
Recently, Schroyens (2010a; 2010b) offered an intriguing alternative hypothesis. He argued that this correlational pattern between truth table task judgments and intelligence cannot be seen as in favour of the ST. He claimed that the truth table task classically used (i.e., the truthbased format of the truth table evaluation task) is flawed and leads to misleading results because of the induction problem: Participants are asked to do something impracticable due to the impossibility of establishing the truth of a universal claim on the basis of a single case. Since they are reluctant to conclude that a singular observation makes a general rule true, participants resist the induced inductive fallacy by judging the false-antecedent-cases as 'irrelevant'. This, as it is claimed, would account for the large number of 'defective' patterns observed in the literature.

It cannot be denied that the original wording of the truth table task (i.e., the case makes the rule true) is rather ambiguous and therefore it cannot be excluded that at least a part of the participants does judge the false-antecedent-cases to be 'irrelevant' in order not to choose the 'true' option, especially when participants have sufficient intelligence to realize that the induction problem might apply. This account (see Schroyens, 2010) therefore must predict that highly able participants are the ones that resist to the inductive fallacy by judging the false-antecedent-cases to be 'undetermined'. The ST on the contrary predicts that the high ability participants will judge the false-antecedent-cases as 'irrelevant'. In order to be able to distinguish between these accounts, in the present experiment participants will be presented with a truth table task in which both the 'irrelevant' and the 'undetermined' option are present.

To test the contrasting predictions of the induction problem account and the ST, we also add a variable 'cognitive ability' to the design of Schroyens (2010). The assessment of participant's intelligence is a significant addition to the existing literature, since it is important to know who are the participants choosing the 'undetermined' option in order to draw conclusions 
with respect to the account of Schroyens (2010a; 2010b) on the one hand and the ST on the other hand.

\section{Experiment 1}

\section{Method}

\section{Participants and Design}

In total 451 first-year university students (17-29 years of age, $M=18.6)$, all unfamiliar with logic, participated in partial fulfilment of course requirements. Participants were run in two groups, respectively completing the four-option truth table task with classical wording $(\mathrm{n}=227)$ and the four-option truth table task with modified wording $(\mathrm{n}=224)$.

\section{Materials and Procedure}

\section{Truth Table Tasks}

In the truth table task paradigm, participants are classically asked to evaluate for each of the four cases (TT, TF, FT and FF) whether that situation 'makes the conditional rule true', 'makes the rule false' or 'is irrelevant with respect to the truth of the rule'. This is the wording that is widely spread in reasoning literature. So in the four-option truth table task with classical wording (TTT4_classical) participants have to judge whether a specific case makes the conditional rule 'true', false', or is 'irrelevant' with respect to the truth of the rule. The fourth option is the undetermined option, stating: 'neither makes the rule false, nor makes the rule irrefutably true - the combination supports the rule, without proving it true or false'. The fouroption truth table task with modified wording (TTT4_modified) is based on Schroyens (2010). Here participants have to judge whether the specific case shows the rule is 'true', 'false' or 'irrelevant'. The forth undetermined option states: 'neither shows the rule is false, nor shows the 
rule is irrefutably true - the combination supports the rule, without proving it true or false'. Since we aimed not only to directly compare both versions of the truth table task but we also wanted our data to be comparable to Schroyens (2010a, 2010b) as well as to results gathered with the classical truth table task, we decided to make use of both task types.

Both groups of participants received the same instructions, appearing on the computer screen and explaining that the purpose of the experiment was to examine how people reason with conditionals. The instructions also contained the description of a machine producing cards with a letter on the front side and a number on the back, always doing so following a certain rule, for example 'If there is an $A$ on the front, there is a 2 on the back of the card'. Participants could read that in the upcoming task they were going to see the content of 16 specific cards produced by that machine, and that per card they had to evaluate the compatibility of the card with the card-producing rule. Participants were then provided with a concrete example of a conditional rule, as well as with an example of the items in the actual task (no correct answers were provided). The instructions were followed by one practice trial. We employed the truth table task with implicit negations, using the negations paradigm ${ }^{1}$ in order to control for the effects of negations and matching bias (see also Evans et al., 2007). No feedback was provided. For each item, the rule followed by one of the 16 situations appeared on the screen. Each item appeared on a different screen and presentation order of the items was randomized. The truth table tasks were constructed with 'E-prime' software (Psychological Software Tools, Pittsburgh, PA) and presented to the participants on individual PCs in a self-paced manner. Responses were given

\footnotetext{
${ }^{1}$ In the negations paradigm, both the antecedent and the consequent of the conditional rule can contain negated constituents. Within participants, four types of conditional rules are used: 'if A then C' (AA), 'if A then not-C' (AN), 'if not-A then $\mathrm{C}$ ' (NA) and finally 'if not-a then not-C' (NN). For each type of rule, participants have to judge all four situations TT, TF, FT and FF.
} 
with the arrow-keys on an AZERTY keyboard. This part of the experiment lasted between 3 and 8 minutes and all parts were administered in Dutch.

Raven's Advanced Progressive Matrices

The variable 'cognitive ability' is assessed by Raven's Advanced Progressive Matrices, measuring participants' inductive or analytic reasoning capacities or fluid intelligence. During the last decades, this has been one of the most widely used instruments by researchers interested in participants' inductive or analytic reasoning capacities or fluid intelligence (Cattell, 1963). Raven's Advanced Progressive Matrices or APM is intended for use with people above average aptitude and designed to reliably differentiate among those in the top $25 \%$ of the population, as we believe is the case for our first-year university students. In the present study a short version of the APM (Bors, \& Stokes, 1998) has been used due to time constraints, since the original version takes at least 40 minutes to be completed. The short version of the APM consists of a selection of 14 items with increasing difficulty drawn from Set 2 of the original APM (item 3, 10, 12, 15, 16, $18,21,22,28,30,31$ and 34). The short version of the APM was administered during the same session than the truth table task. For all 14 items, participants had to indicate which was the missing segment required to complete a $3 \times 3$ matrix, which takes about 20 minutes. Scores range from 0 to 12 as the first two items are conceived as 'practice trials'.

\section{Results}

First of all, T-tests revealed no difference at all between both wording-types of the truth table task (see Table 1). For none of the computed indices a difference was revealed between the four-option truth table task with classical wording and the four-option version with adjusted wording. Moreover, mean scores on the APM did not differ, so both groups are comparable regarding intelligence. The absence of any difference allows us to merge the data of the 
participants in both task-wording groups, so in the analyses below we will make abstraction of this variable and always describe the whole group of 451 participants ${ }^{2}$.

\section{"(Table 1)"}

With respect to participants' classifications of the truth table cases, we observe an overall tendency of classification in conformity with a three-valued or a four-valued truth table pattern, i.e., 'true' for the TT case, 'false' for the TF case and 'irrelevant' or 'undetermined' for the falseantecedent-cases FT and FF (see Table 2). For the false-antecedent-cases, the 'undetermined' option is chosen in $32 \%$ and 'irrelevant' in $35 \%$ of the judgments.

\section{"(Table 2)"}

Considering the relation between participants' truth table classifications and intelligence, we computed four different false antecedent indices: per participant the sum of the falseantecedent-cases judged respectively as 'true' $\left(F A_{-} T-I\right)$, as 'false' $\left(F A \_F-I\right)$, as 'irrelevant' $\left(F A \_I-I\right)$ and finally as 'undetermined' (FA_U-I). All four FA-Indices range from 0 to 8 . In addition to the false antecedent indices, we computed three more indexes. According to the ST, participants should judge the TT case as 'true', the TF case as 'false' and both the FT and the FF case as 'irrelevant'. We combined these judgments into the suppositional theory index (ST-I), ranging from 0 to 16 per participant. Moreover, we calculated the mental models theory index or material implication index (MI-I), summing per participant the number of TT cases judged as 'true', TF cases as 'false', and FT and FF cases as 'true' (range 0-16). Finally we computed a

\footnotetext{
${ }^{2}$ The fact that the rather subtle manipulation of the wording factor did not yield mean differences does not necessarily imply that correlations are unaffected. Therefore, correlations were also computed for each wordinggroup separately. The correlational pattern however was identical so for the sake of simplicity only the global correlation analysis, conjoining both wording groups, is reported.
} 
conjunctivity index (Conj-I) with a range from 0 to 16 . This is the classification pattern in which only the TT case is judged as 'true' and the three other cases as 'false'.

First of all a negative ${ }^{3}$ correlation (-.21) was revealed between the APM and the ConjIndex. Secondly, there was a positive correlation between the APM and the ST-Index (.19) and a negative correlation between the APM and the MI-I (-.19). Finally, regarding the four different false antecedent indices, the following correlations between the APM on the one hand and the false-antecedent-cases on the other hand are observed: A negative correlation for the falseantecedent-cases judged as 'false' (-.25) and as 'true' (-.27) and a positive correlation for the false-antecedent-cases classified as 'irrelevant' (.21) and as 'undetermined' (.11).

\section{Discussion}

Whereas the observation of 'defective' answer patterns can be seen as in favour of the ST (Evans et al., 2007; Sevenants et al., 2008; 2010), Schroyens (2010a; 2010b) claims that participants judge the false-antecedent-cases as 'irrelevant' not due to a suppositional representation but instead because of the induction problem they want to resist. In the present experiment the impact of the induction problem on the results of the classical truth table task is tested by inspecting the correlation between the observed answer patterns and intelligence. The account of Schroyens must predict 'undetermined' instead of 'true' judgments by the highintelligence participants, whereas the ST predicts these highly able participants to judge the falseantecedent-cases as 'irrelevant'.

With respect to false-antecedent-cases, the 'undetermined' option is chosen in $32 \%$ of the judgments. This fairly high percentage of 'undetermined' judgments might indicate that when they have the opportunity to do so, one third of the participants choose to resist to the induction

\footnotetext{
${ }^{3}$ All reported correlations are significant at the .001 level, except for the correlation between APM and 'undetermined', which is significant at the .05 level.
} 
problem by judging the false-antecedent-cases to be 'undetermined' with respect to the conditional rule. Moreover, a small but positive correlation exists between the APM and the judgment of false-antecedent-cases as 'undetermined'. This is evidence for the induction problem.

The induction problem account however is subject to some important considerations. Firstly, participants do not hesitate to judge the TT case as 'true' instead of as 'undetermined' (for 'irrelevant', see also Evans, 2007; Sevenants et al., 2011; Sevenants, Schroyens, Dieussaert \& Schaeken, 2008), even when this case is equally well subject to the inductive fallacy as the other truth table cases. A MMT response to this objection would be that the TT case is represented in the initial model and therefore the default answer which is hard to resist, but this cannot account for the fact that so little 'undetermined' judgments are observed for this TT case. Secondly, even with the fourth 'undetermined' option available, in $35 \%$ of the classifications the false-antecedent-cases are still judged as 'irrelevant', suggesting a suppositional representation of conditionals. This high number of 'defective' answer patterns is also observed in experiments that do not make use of the wording 'make true' and therefore do not induce the inductive fallacy. This is the case for experiments where 'conform to' is used instead (for an overview, see Schroyens, 2010a) and also in three-option possibilities tasks (Sevenants et al., 2008; 2011). Thirdly, a positive correlation between the classification of false-antecedent-cases as 'irrelevant' and the intelligence measure is observed, even with the fourth option available. This is evidence in support of the ST predicting a positive correlation between intelligence and the number of false-antecedent-cases classified as 'irrelevant'. Finally, the equal number of 'irrelevant' and 'undetermined' judgments can also be explained by people's tendency to use all the response options available to them. 
In sum, the truth table judgments and the observed correlations between these judgments and intelligence support a suppositional processing of conditionals, but don't exclude that a part of the observed 'defective' answer patterns observed in classical truth table task experiments might be due to the induction problem. By means of some methodological refinements and participants' justifications of their judgments, we aim to provide a direct test of the induction problem account in a second experiment.

\section{Experiment 2}

In Experiment 1 participants were presented with truth table tasks with four answer options, in which the fourth 'undetermined' option was the following: 'neither shows the rule is false, nor shows the rule is irrefutably true - the combination supports the rule, without proving it true or false'. It was observed that this option was chosen in about one third of the falseantecedent judgments. Instead of providing evidence for the induction problem account, it might also be the case that this high number of 'undetermined' responses points to a problem with the psychometric soundness of the response format. Indeed, the 'undetermined' option consists of a 22-word sentence, which is considerably longer than the other options. Such a marked option is likely to introduce a bias in its favour and might cause a power of suggestion to the effect which participants might never have envisaged otherwise. Therefore, in this second experiment we will use a fourth 'undetermined' option that has the same length as the 'irrelevant' option.

Secondly, in Experiment 1 the negations paradigm was used in order to control for matching bias. However, the use of materials that include negated antecedents and consequents introduces a cognitive load linked with the problems of the processing of negations. In order to gain insight in participants' understanding of the answer options without being subject to an extra 
cognitive load, in Experiment 2 no negations will be used in the conditional rule. More concretely, participants will only be presented with the 'if $A$ then $C$ ' rule (AA).

Finally, an important question raised by Experiment 1 is whether participants really make a systematic distinction between 'irrelevant' and 'undetermined'. For a suppositional conditional, saying that the rule is 'undetermined' by a given case is not that different to claiming that a case is 'irrelevant' with respect to a rule - they both indicate that a mental simulation cannot be engaged. In order to shed light on this issue, in the present experiment we ask participants for a justification of their judgments. This will provide us with a clear picture of how participants exactly understand the answer options, which is indispensable in order to draw conclusions about the underlying reason for their judgments.

\section{Participants and Design}

Sixty-seven first-year high-school students, unfamiliar with logic, participated in partial fulfilment of course requirements (17-25 years of age, $\mathrm{M}=18.3)$.All participants were presented with the same four-option version of the truth table task.

\section{Materials and Procedure}

\section{Truth Table Tasks}

Participants are asked to evaluate for each of the four cases (TT, TF, FT and FF) whether that situation 'makes the rule true', 'makes the rule false', 'is irrelevant with respect to the truth of the rule' or 'cannot make the rule true nor make it false'. So in the present version of the fouroption truth table task, the 'irrelevant' and the 'undetermined' option are equally lengthy. Since the negations paradigm will not be used, participants are only presented with one conditional rule (AA; without negations) for which they have to judge the four situations. After each judgment, 
participants have to write down a justification for that judgment. The experiment is presented in printed booklets consisting of five pages, with on the first page the same instructions as in Experiment 1 (except for the justifications). On each of the other four pages, the conditional rule along with one situation is printed. Next to the four answer options, a text box for the justifications is provided on each page. In the four situations implicit negations were used, as was the case in Experiment 1. The experiment was presented in Dutch and lasted between 3 and 7 minutes.

\section{Results}

Regarding participants' judgments (see Table 3) of the true-antecedent cases, the TT case is classified as 'true' by all participants and the TF case as 'false' by $95 \%$ of the participants. With respect to the false-antecedent-cases, FT is judged as 'undetermined' by half of the participants, as 'false' by more than one third and as 'irrelevant' by one fourth of the participants. The FF caseis judged as 'irrelevant' by half of the participants and both the 'undetermined' and the 'false' options are chosen by one fourth of the participants.

\section{"(Table 3)"}

With respect to the categorisation of the justifications, there was only one rater since all justifications could be classified unambiguously. Participants almost literally paraphrased the justifications as discussed below. All participants wrote down that the TT case makes the rule 'true' "because the situation is exactly what the rule describes". The justification for judging the $\mathrm{TF}$ case as making the rule 'false' is "the situation does not correspond to what the rule prescribes". This is also the justification that is given for $69 \%$ of the "false' judgments of the false-antecedent-cases. $31 \%$ of the 'false' judgments of these cases is explicitly accounted for in 
terms of a "mismatch" between the rule and the situation. Regarding the 'irrelevant' justifications of the false-antecedent-cases, $6 \%$ of these judgments is accounted for as "one cannot know", $35 \%$ as "it is possible but not necessary the case that the situation makes the rule true" and 59\% in terms of "the letters and the numbers of the rule and the situation are not the same so the situation has nothing to do with the rule". Finally, one single participant accounts for his 'irrelevant' judgments of the false-antecedent-cases by referring to the induction problem, writing down that "one situation cannot make a rule true". The same type of justifications is observed for the 'undetermined' judgments: In $23 \%$ of these classification, participants refers to "one cannot know", in $35 \%$ to "possible but not necessary" and finally in $43 \%$ of the 'undetermined' options to "the situation having nothing to do with the rule because of differing letters-numbers".

\section{Discussion}

All participants judge the TT case as 'true' and the TF case as 'false'. The total number of 'irrelevant' and 'undetermined' classifications is the same, but 'irrelevant' is more frequently observed for the FF case and 'undetermined' for the FT case. The global pattern of results observed in this second experiment therefore corresponds to the judgments observed in Experiment 1 . This shows that the reason for choosing the 'undetermined' option is not a bias in favour of this option due to its length, as we could not exclude based on Experiment 1: When the number of words of the 'irrelevant' and the 'undetermined' option is comparable, the latter is chosen equally frequent than when the 'undetermined' option was significantly longer than the 'irrelevant' option. Likewise, we can conclude that the results observed in Experiment 1 (i.e., the high number of 'irrelevant' and 'undetermined' judgments) was not due to the use of the negations paradigm and thus to an extra cognitive load because of the presence of negations in 
the conditional rule. Both in Experiment 1 with the negations paradigm and in Experiment 2 without the negations paradigm, 'irrelevant' and 'undetermined' make up the dominant classification for the false-antecedent-cases.

Inspecting the justifications, only one participant refers to the induction problem as the reason for choosing the 'irrelevant' option. Almost all participants refer to the possibility that the situation makes the rule true or to the fact that the situation has nothing to say about the rule. This is an explanation that is closely related to the 'truth value gap' justification for 'irrelevant' answers of the ST (see also Sevenants et al., 2008).

From this we conclude that participants do not seem to make a systematic distinction between 'irrelevant' and 'undetermined', but that they do provide a meaningful justification for their judgments, which is not related to the induction problem. This conclusion is also supported by the observation that in the present experiment not a single participant hesitates to give a 'true' judgment for the TT case, even though they could have chosen both the 'irrelevant' or the 'undetermined' to resist to the inductive fallacy.

\section{General Discussion}

In two experiments we examine the claim of Schroyens (2010a; 2010b) that not a suppositional processing of conditionals (Evans \& Over, 2004, Evans et al., 2007) but rather the induction problem is the main reason for participants to judge false-antecedent-cases as 'irrelevant' in the truth table task paradigm. We observe that the majority of false-antecedentcases are judged both as 'irrelevant' and 'undetermined', especially by the most intelligent participants, as is shown by a correlation analysis (Experiment 1). This could suggest that these participants wish to resist the inductive fallacy by choosing one of these options. The observation however that all participants judge the TT case as 'true', together with the justifications in which 
only one participants refers to the induction problem (Experiment 2), leads us to the conclusion that the judgments of false-antecedent-cases as 'irrelevant' can be attributed to a suppositional processing of conditionals.

In sum, with the present study we show that the dominant 'irrelevant' or 'undetermined' judgments of false-antecedent-cases in truth table task experiments are due to a suppositional representation of conditionals. Firstly, these options are chosen by the most intelligent participants and secondly the justifications show that the induction problem is not the underlying reason. These observations are problematic for the MMT, which would predict the most intelligent participants to judge the false-antecedent-cases as 'true'.

\section{References}

Barrouillet, P., Gauffroy, C., \& Lecas, J. F. (2008). Mental models and the suppositional account of conditionals. Psychological Review, 115, 760-772.

Bors, D. A., \& Stokes, T. L. (1998). Raven's advanced progressive matrices: Norms for first-year university students and the development of a short form. Educational and Psychological Measurement, 58, 382-398.

de Finetti, B. (1967). Sur quelques conventions qui semblent utiles. [On some conventions that seem useful]. Revue Roumaine de Mathématiques Pures et Appliquées, xii, 1227-1234.

Evans, J. St. B. T., Over, D., (2004). If. Oxford University Press.

Evans, J. St. B. T., Handley, S. H., Neilens, H., \& Over, D. E. (2007). Thinking about conditionals: A study of individual differences. Memory \& Cognition, 35, 1722-1784.

Frederick, S. (2005). Cognitive reflection and decision making. Journal of Economic Perspectives, 19, 25-42.

Johnson-Laird, P. N., \& Byrne, R. M. J. (1991). Deduction. Hillsdale, NJ: Erlbaum. 
Johnson-Laird, P. N., \& Byrne, R. M. J. (2002). Conditionals: A theory of meaning, pragmatics, and inference. Psychological Review, 109, 646-678.

Politzer, G., Over, D., \& Baratgin, J. (2010). Betting on conditionals. Thinking and Reasoning, 3, 172-197.

Ramsey, F. P. (1990). General propositions and causality (original publication, 1931). In D. H. Mellor (Ed.), Philosophical Papers (pp. 145-163). Cambridge: Cambridge University Press.

Schroyens, W. (2010a). Mistaking the instance for the rule: A critical analysis of the truth-table evaluation paradigm. Quarterly Journal of Experimental Psychology, 63, 246-259.

Schroyens, W. (2010b). A meta-analytic review of thinking about what is true, possible, and irrelevant in reasoning from or reasoning about conditional propositions. European Journal of Cognitive Psychology, 22, 897921.

Sevenants, A., Dieussaert, K., \& Schaeken, W. (2011). Truth table tasks: Irrelevance and cognitive ability. Thinking \& Reasoning? 17, 213- 246.

Sevenants, A., Schroyens, W., Dieussaert, K., Schaeken, W., \& d'Ydewalle, G. (2008). Truth table tasks: The relevance of irrelevant. Thinking \& Reasoning, 14, 409-433. 